CUBO A Mathematical Journal

Vol.12, $N^{\underline{O}} 01$, (133-148). March 2010

\title{
Well-Posedness Results for Anisotropic Nonlinear Elliptic Equations with Variable Exponent and $L^{1}$-Data
}

\author{
STANISLAS OUARO \\ Laboratoire d'Analyse Mathématique des Equations (LAME), \\ UFR. Sciences Exactes et Appliquées, Université de Ouagadougou \\ 03 BP 7021 Ouaga 03 \\ Ouagadougou, Burkina Faso \\ email: souaro@univ-ouaga.bf
}

\begin{abstract}
We study the anisotropic boundary value problem $-\sum_{i=1}^{N} \frac{\partial}{\partial x_{i}} a_{i}\left(x, \frac{\partial}{\partial x_{i}} u\right)=f$ in $\Omega, u=0$ on $\partial \Omega$, where $\Omega$ is a smooth open bounded domain in $\mathbb{R}^{N}(N \geq 3)$ and $f \in L^{1}(\Omega)$. We prove the existence and uniqueness of an entropy solution for this problem.
\end{abstract}

\section{RESUMEN}

Estudiamos el problema de valores en la frontera anisotropico $-\sum_{i=1}^{N} \frac{\partial}{\partial x_{i}} a_{i}\left(x, \frac{\partial}{\partial x_{i}} u\right)=f$ en $\Omega, u=0$ sobre $\partial \Omega$, donde $\Omega$ es un dominio abierto suave do $\mathbb{R}^{N}(N \geq 3)$ y $f \in L^{1}(\Omega)$. Proveamos la existencia y unicidad de una solución de entropía para este problema.

Key words and phrases: Anisotropic; variable exponent; entropy solution; electrorheological fluids.

Math. Subj. Class.: 35J20, 35J25, 35D30, 35B38, 35J60. 


\section{Introduction}

Let $\Omega$ be an open bounded domain of $\mathbb{R}^{N}(N \geq 3)$ with smooth boundary. Our aim is to prove the existence and uniqueness of an entropy solution to the anisotropic nonlinear elliptic problem

$$
\left\{\begin{array}{l}
-\sum_{i=1}^{N} \frac{\partial}{\partial x_{i}} a_{i}\left(x, \frac{\partial}{\partial x_{i}} u\right)=f \text { in } \Omega \\
u=0 \text { on } \partial \Omega,
\end{array}\right.
$$

where the right-hand side $f \in L^{1}(\Omega)$.

We assume that for $i=1, \ldots, N$ the function $a_{i}: \Omega \times \mathbb{R} \rightarrow \mathbb{R}$ is Carathéodory and satisfies the following conditions: $a_{i}(x, \xi)$ is the continuous derivative with respect to $\xi$ of the mapping $A_{i}: \Omega \times \mathbb{R} \rightarrow \mathbb{R}, A_{i}=A_{i}(x, \xi)$, i.e. $a_{i}(x, \xi)=\frac{\partial}{\partial \xi} A_{i}(x, \xi)$ such that the following equality and inequalities holds

$$
A_{i}(x, 0)=0,
$$

for almost every $x \in \Omega$.

There exists a positive constant $C_{1}$ such that

$$
\left|a_{i}(x, \xi)\right| \leq C_{1}\left(j_{i}(x)+|\xi|^{p_{i}(x)-1}\right)
$$

for almost every $x \in \Omega$ and for every $\xi \in \mathbb{R}$, where $j_{i}$ is a nonnegative function in $L^{p_{i}^{\prime}(.)}(\Omega)$, with $1 / p_{i}(x)+1 / p_{i}^{\prime}(x)=1$.

There exists a positive constant $C_{2}$ such that

$$
\left(a_{i}(x, \xi)-a_{i}(x, \eta)\right) \cdot(\xi-\eta) \geq \begin{cases}C_{2}|\xi-\eta|^{p_{i}(x)} & \text { if }|\xi-\eta| \geq 1 \\ C_{2}|\xi-\eta|^{p_{i}^{-}} & \text {if }|\xi-\eta|<1,\end{cases}
$$

for almost every $x \in \Omega$ and for every $\xi, \eta \in \mathbb{R}$, with $\xi \neq \eta$,

and

$$
|\xi|^{p_{i}(x)} \leq a_{i}(x, \xi) \cdot \xi \leq p_{i}(x) A_{i}(x, \xi)
$$

for almost every $x \in \Omega$ and for every $\xi \in \mathbb{R}$.

We also assume that the variable exponent $p_{i}():. \bar{\Omega} \rightarrow[2, N)$ are continuous functions for all $i=1, \ldots, N$ such that:

$$
\frac{\bar{p}(N-1)}{N(\bar{p}-1)}<p_{i}^{-}<\frac{\bar{p}(N-1)}{N-\bar{p}}, \quad \sum_{i=1}^{N} \frac{1}{p_{i}^{-}}>1 \text { and } \frac{p_{i}^{+}-p_{i}^{-}-1}{p_{i}^{-}}<\frac{\bar{p}-N}{\bar{p}(N-1)},
$$

where $\frac{1}{\bar{p}}=\frac{1}{N} \sum_{i=1}^{N} \frac{1}{p_{i}^{-}}, \quad p_{i}^{-}:=e s s \inf _{x \in \Omega} p_{i}(x)$ and $p_{i}^{+}:=e s s \sup _{x \in \Omega} p_{i}(x)$.

We introduce the numbers

$$
q=\frac{N(\bar{p}-1)}{N-1}, q^{*}=\frac{N q}{N-q}=\frac{N(\bar{p}-1)}{N-\bar{p}} .
$$


A prototype example that is covered by our assumptions is the following anisotropic $\left(p_{1}(),. \ldots, p_{N}().\right)$ harmonic equation:

Set $A_{i}(x, \xi)=\left(1 / p_{i}(x)\right)|\xi|^{p_{i}(x)}, a_{i}(x, \xi)=|\xi|^{p_{i}(x)-2} \xi$ where $p_{i}(x) \geq 2$. Then we get the following equation:

$$
-\sum_{i=1}^{N} \frac{\partial}{\partial x_{i}}\left(\left|\frac{\partial}{\partial x_{i}} u\right|^{p_{i}(x)-2} \frac{\partial}{\partial x_{i}} u\right)=f
$$

which, in the particular case when $p_{i}=p$ for any $i \in\{1, \ldots, N\}$ is the anisotropic $p($.$) -Laplace$ equation.

For the proof of existence of entropy solutions of (1.1), we follow [2] and derive a priori estimates for the approximated solutions $u_{n}$ and the partial derivatives $\frac{\partial u_{n}}{\partial x_{i}}$ in the Marcinkiewicz spaces $\mathcal{M}^{q^{*}}$ and $\mathcal{M}^{p_{i}^{-}} q / \bar{p}$ respectively (see section 2 or [2] for definition of Marcinkiewicz spaces).

The study of nonlinear elliptic equations involving the $p$-Laplace operator is based on the theory of standard Sobolev spaces $W^{m, p}(\Omega)$ in order to find weak solutions. For the nonhomogeneous $p($.$) -Laplace operators, the natural setting for this approach is the use of the variable exponent$ Lebesgue and Sobolev spaces $L^{p(\cdot)}(\Omega)$ and $W^{m, p(.)}(\Omega)$.

Variable exponent Lebesgue spaces appeared in the literature for the first time in a 1931 article by Orlicz[21]. After [21], Orlicz abandoned the study of variable exponent spaces to concentrate on the theory of function spaces that now bears his name (Orlicz spaces). After Orlicz's work (cf. [21]), H. Hudzik [14], and J. Musielak [20] investigated the variable exponent Sobolev spaces.

Variable exponent Lebesgue spaces on the real line have been independently developed by Russian researchers, notably Sharapudinov[26] and Tsenov [27]. The next major step in the investigation of variable exponent Lebesgue and Sobolev spaces was the comprehensive paper by O. Kovacik and J. Rakosnik in the early 90's [16]. This paper established many of basic properties of Lebesgue and Sobolev spaces with variables exponent. Variable Sobolev spaces have been used in the last decades to model various phenomena. In [5], Chen, Levine and Rao proposed a framework for image restoration based on a Laplacian variable exponent. Another application which uses nonhomogeneous Laplace operators is related to the modelling of electrorheological fluids. The first major discovery in electrorheological fluids was due to Willis Winslow in 1949 (cf. [28]). These fluids have the interesting property that their viscosity depends on the electric field in the fluid. They can raise the viscosity by as much as five orders of magnitude. This phenomenon is known as the Winslow effect. For some technical applications, consult Pfeiffer et al [22]. Electrorheological fluids have been used in robotics and space technology. The experimental research has been done mainly in the USA, for instance in NASA laboratories. For more information on properties, modelling and the application of variable exponent spaces to these fluids, we refer to Diening [6], Rajagopal and Ruzicka [22], and Ruzicka [24].

In this paper, the operator involved in (1.1) is more general than the $p($.$) -Laplace operator. Thus,$ the variable exponent Sobolev space $W^{1, p(.)}(\Omega)$ is not adequate to study nonlinear problems of this type. This leads us to seek entropy solutions for problems (1.1) in a more general variable exponent Sobolev space which was introduced for the first time by Mihaïlescu et al [18]. 
As the right-hand side in (1.1) is in $L^{1}(\Omega)$, the suitable notion of solution is a notion of entropy solution (cf. [3]).

The remaining part of this paper is organized as follows: Section 2 is devoted to mathematical preliminaries including, among other things, a brief discussion of variable exponent Lebesgue, Sobolev, anisotropic spaces and Marcinkiewicz spaces. Existence of weak energy solution for (1.1) where $f \in L^{\infty}(\Omega)$ was proved in [14]; We will also briefly recall the results of [14] in section 2 . The main existence and uniqueness result is stated and proved in section 3 .

\section{Mathematical Preliminaries}

In this section, we define Lebesgue, Sobolev and anisotropic spaces with variable exponent and give some of their properties. Roughly speaking, anistropic Lebesgue and Sobolev spaces are functional spaces of Lebesgue's and Sobolev's type in which different space directions have different roles.

Given a measurable function $p():. \Omega \rightarrow[1, \infty)$. We define the Lebesgue space with variable exponent $L^{p(.)}(\Omega)$ as the set of all measurable function $u: \Omega \rightarrow \mathbb{R}$ for which the convex modular

$$
\rho_{p(.)}(u):=\int_{\Omega}|u|^{p(x)} d x
$$

is finite. If the exponent is bounded, i.e., if $p_{+}<\infty$, then the expression

$$
|u|_{p(.)}:=\inf \left\{\lambda>0: \rho_{p(.)}(u / \lambda) \leq 1\right\}
$$

defines a norm in $L^{p(\cdot)}(\Omega)$, called the Luxembourg norm. The space $\left(L^{p(\cdot)}(\Omega),|\cdot|_{p(.)}\right)$ is a separable Banach space. Moreover, if $p_{-}>1$, then $L^{p(\cdot)}(\Omega)$ is uniformly convex, hence reflexive, and its dual space is isomorphic to $L^{p^{\prime}(.)}(\Omega)$, where $\frac{1}{p(x)}+\frac{1}{p^{\prime}(x)}=1$. Finally, we have the Hölder type inequality:

$$
\left|\int_{\Omega} u v d x\right| \leq\left(\frac{1}{p_{-}}+\frac{1}{p_{-}^{\prime}}\right)|u|_{p(.)}|v|_{p^{\prime}(.)},
$$

for all $u \in L^{p(\cdot)}(\Omega)$ and $v \in L^{p^{\prime}(\cdot)}(\Omega)$.

Now, let

$$
W^{1, p(.)}(\Omega):=\left\{u \in L^{p(.)}(\Omega):|\nabla u| \in L^{p(.)}(\Omega)\right\},
$$

which is a Banach space equipped with the norm

$$
\|u\|_{1, p(.)}:=|u|_{p(.)}+|\nabla u|_{p(.)} .
$$

An important role in manipulating the generalized Lebesgue-Sobolev spaces is played by the modular $\rho_{p(.)}$ of the space $L^{p(.)}(\Omega)$.

Next, we define $W_{0}^{1, p(.)}(\Omega)$ as the closure of $C_{0}^{\infty}(\Omega)$ in $W^{1, p(.)}(\Omega)$ under the norm $\|u\|_{1, p(.)}$.

Set $C_{+}(\bar{\Omega})=\left\{p(.) \in C(\bar{\Omega}): \min _{x \in \bar{\Omega}} p(x)>1\right\}$.

Furthermore, if $p(.) \in C_{+}(\bar{\Omega})$ is logarithmic Hölder continuous, then $C_{0}^{\infty}(\Omega)$ is dense in $W_{0}^{1, p(.)}(\Omega)$, 
that is $H_{0}^{1, p(.)}(\Omega)=W_{0}^{1, p(.)}(\Omega)$ (cf. [13]). Since $\Omega$ is an open bounded set and $p(.) \in C_{+}(\bar{\Omega})$ is logarithmic Hölder, the $p($.$) -Poincaré inequality$

$$
|u|_{p} \leq C|\nabla u|_{p(.)}
$$

holds for all $u \in W_{0}^{1, p(.)}(\Omega)$, where $C$ depends on $p,|\Omega|, \operatorname{diam}(\Omega)$ and $N$ (see [13]), and so

$$
\|u\|:=|\nabla u|_{p(.)},
$$

is an equivalent norm in $W_{0}^{1, p(.)}(\Omega)$. Of course also the norm

$$
\|u\|_{p(.)}:=\sum_{i=1}^{N}\left|\frac{\partial}{\partial x_{i}} u\right|_{p(.)}
$$

is an equivalent norm in $W_{0}^{1, p(.)}(\Omega)$. Hence the space $W_{0}^{1, p(.)}(\Omega)$ is a separable and reflexive Banach space.

Let us present a natural generalization of the variable exponent Sobolev space $W_{0}^{1, p(.)}(\Omega)(\mathrm{cf} .[18])$ that will enable us to study the problem (1.1) with sufficient accuracy.

First of all, we denote by $\vec{p}():. \bar{\Omega} \rightarrow \mathbb{R}^{N}$ the vectorial function $\vec{p}=\left(p_{1}(),. \ldots, p_{N}().\right)$. The anisotropic variable exponent Sobolev space $W_{0}^{1, \vec{p}(.)}(\Omega)$ is defined as the closure of $C_{0}^{\infty}(\Omega)$ with respect to the norm

$$
\|u\|_{\vec{p}_{(.)}}:=\sum_{i=1}^{N}\left|\frac{\partial}{\partial x_{i}} u\right|_{p_{i}(.)} .
$$

The space $\left(W_{0}^{1, \vec{p}(.)}(\Omega),\|u\|_{\vec{p}(.)}\right)$ is a reflexive Banach space (cf. [18]).

Let us introduce the following notations:

$$
\begin{gathered}
\vec{P}_{+}=\left(p_{1}^{+}, \ldots, p_{N}^{+}\right), \vec{P}_{-}=\left(p_{1}^{-}, \ldots, p_{N}^{-}\right), \\
P_{+}^{+}=\max \left\{p_{1}^{+}, \ldots, p_{N}^{+}\right\}, P_{-}^{+}=\max \left\{p_{1}^{-}, \ldots, p_{N}^{-}\right\}, P_{-}^{-}=\min \left\{p_{1}^{-}, \ldots, p_{N}^{-}\right\},
\end{gathered}
$$

and

$$
P_{-}^{*}=\frac{N}{\sum_{i=1}^{N} \frac{1}{p_{i}^{-}}-1}, P_{-, \infty}=\max \left\{P_{-}^{+}, P_{-}^{*}\right\}
$$

We have the following result (cf. [18]):

Theorem 2.1. Assume $\Omega \subset \mathbb{R}^{N}(N \geq 3)$ is a bounded domain with smooth boundary. Assume relation (1.6) is fulfilled. For any $q \in C(\bar{\Omega})$ verifying

$$
1<q(x)<P_{-, \infty} \text { for all } x \in \bar{\Omega}
$$


then the embedding

$$
W_{0}^{1, \vec{p}(\cdot)}(\Omega) \hookrightarrow L^{q(\cdot)}(\Omega)
$$

is continuous and compact.

We Also recall the result of the study of problem (1.1) for the right-hand side $f \in L^{\infty}(\Omega)$ (cf. [15]). We first recall the definition of weak energy solution of (1.1) for the right-hand side more regular i.e $f \in L^{\infty}(\Omega)$.

Definition 2.2. Let $f \in L^{\infty}(\Omega)$; a weak energy solution of (1.1) is a function $u \in W_{0}^{1, \vec{p}(.)}(\Omega)$ such that

$$
\int_{\Omega} \sum_{i=1}^{N} a_{i}\left(x, \frac{\partial}{\partial x_{i}} u\right) \cdot \frac{\partial}{\partial x_{i}} \varphi d x=\int_{\Omega} f(x) \varphi d x, \text { for all } \varphi \in W_{0}^{1, \vec{p}(.)}(\Omega) .
$$

We have proved among other results in [15] the following Theorem

Theorem 2.3. Assume (1.2)-(1.6) and $f \in L^{\infty}(\Omega)$. Then there exists a unique weak energy solution of (1.1).

Finally, in this paper, we will use the Marcinkiewicz spaces $\mathcal{M}^{q}(\Omega)(1<q<\infty)$ with constant exponent. Note that the Marcinkiewicz spaces $\mathcal{M}^{q(.)}(\Omega)$ in the variable exponent setting was introduced for the first time by Sanchon and Urbano (see [25]).

Marcinkiewicz spaces $\mathcal{M}^{q}(\Omega)(1<q<\infty)$ contain the measurable functions $g: \Omega \rightarrow \mathbb{R}$ for which the distribution function

$$
\lambda_{g}(\gamma)=|\{x \in \Omega:|g(x)|>\gamma\}|, \gamma \geq 0,
$$

satisfies an estimate of the form

$$
\lambda_{g}(\gamma) \leq C \gamma^{-q}, \text { for some finite constant } C>0 .
$$

The space $\mathcal{M}^{q}(\Omega)$ is a Banach space under the norm

$$
\|g\|_{\mathcal{M}^{q}(\Omega)}^{*}=\sup _{t>0} t^{1 / q}\left(\frac{1}{t} \int_{0}^{t} g^{*}(s) d s\right)
$$

where $g^{*}$ denotes the nonincreasing rearrangement of $f$ :

$$
g^{*}(t)=\inf \left\{\gamma>0: \lambda_{g}(\gamma) \leq t\right\}
$$

We will use the following pseudo norm

$$
\|g\|_{\mathcal{M}^{q}(\Omega)}=\inf \left\{C: \lambda_{g}(\gamma) \leq C \gamma^{-q}, \forall \gamma>0\right\}
$$

which is equivalent to the norm $\|g\|_{\mathcal{M}^{q}(\Omega)}^{*}$ (see [2]).

We have the following Lemma (for the proof, see [2, proof of Lemma 2.2]).

Lemma 2.4. Let $g$ be a nonnegative function in $W_{0}^{1, \vec{P}_{-}}(\Omega)$. Assume $\bar{p}<N$, and that there exists a constant $c$ such that

$$
\sum_{i=1}^{N} \int_{\{|g| \leq \gamma\}}\left|\frac{\partial g}{\partial x_{i}}\right|^{p_{i}^{-}} d x \leq c(\gamma+1), \forall \gamma>0
$$


Then there exists a constant $C$, depending on $c$, such that

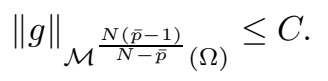

\section{Existence and Uniqueness of Entropy Solution}

In this section, we study the problem (1.1) for a right-hand side $f \in L^{1}(\Omega)$. In the $L^{1}$ setting, the suitable notion of solution for the study of (1.1) is the notion of entropy solution (cf. [3]).

We first define the troncation function $T_{t}$ by $T_{t}(s):=\max \{-t, \min \{t, s\}\}$.

Definition 3.1. A measurable function $u$ is an entropy solution to problem (1.1) if, for every $t>0, T_{t}(u) \in W_{0}^{1, \vec{p}(.)}(\Omega)$ and

$$
\int_{\Omega} \sum_{i=1}^{N} a_{i}\left(x, \frac{\partial}{\partial x_{i}} u\right) \cdot \frac{\partial}{\partial x_{i}} T_{t}(u-\varphi) d x \leq \int_{\Omega} f(x) T_{t}(u-\varphi) d x
$$

for all $\varphi \in W_{0}^{1, \vec{p}(.)}(\Omega) \cap L^{\infty}(\Omega)$.

Remark 3.2. A function $u$ such that $T_{t}(u) \in W_{0}^{1, \vec{p}(.)}(\Omega)$ for all $t>0$ does not necessarily belong in $W_{0}^{1,1}(\Omega)$. However, it is possible to define its weak gradient, still denoted by $\nabla u$.

Our main result in this section is the following:

Theorem 3.3. Assume (1.2)-(1.6) and $f \in L^{1}(\Omega)$. Then there exists a unique entropy solution u to problem (1.1).

\section{Proof.}

The proof of this Theorem will be done in three steps.

$*$ Step 1. A priori estimates. We start with the existence of the weak gradient for every measurable function $u$ such that $T_{t}(u) \in W_{0}^{1, \vec{p}(.)}(\Omega)$ for all $t>0$.

Proposition 3.4. If $u$ is a measurable function such that $T_{t}(u) \in W_{0}^{1, \vec{p}(.)}(\Omega)$ for all $t>0$, then there exists a unique measurable function $v: \Omega \rightarrow \mathbb{R}^{N}$ such that

$$
v \chi_{\{|u|<t\}}=\nabla T_{t}(u) \text { for a.e. } x \in \Omega \text {, and for all } t>0,
$$

where $\chi_{A}$ denotes the characteristic function of a measurable set A. Moreover, if u belongs to $W_{0}^{1,1}(\Omega)$, then $v$ coincides with the standard distributional gradient of $u$.

Proof. As $T_{t}(u) \in W_{0}^{1, \vec{p}^{(.)}}(\Omega) \hookrightarrow W_{0}^{1, \vec{P}_{-}}(\Omega) \subset W_{0}^{1,1}(\Omega)$ for all $t>0$ since $1<p_{i}^{-}$for all $i=1, \ldots, N$, then by Theorem 1.5 in [1], the result follows.

Proposition 3.5. Assume (1.2)-(1.6) and $f \in L^{1}(\Omega)$. Let $u$ be an entropy solution of (1.1). If there exists a positive constant $M$ such that

$$
\sum_{i=1}^{N} \int_{\{|u|>t\}} t^{q_{i}(x)} d x \leq M, \text { for all } t>0,
$$

then

$$
\sum_{i=1}^{N} \int_{\left\{\left|\frac{\partial}{\partial x_{i}} u\right|^{\alpha_{i}(\cdot)}>t\right\}} t^{q_{i}(x)} d x \leq\|f\|_{1}+M \text { for all } t>0
$$


where $\alpha_{i}()=.p_{i}() /.\left(q_{i}()+1.\right)$, for all $i=1, \ldots, N$.

Proof. Take $\varphi=0$ in (3.1), we have

$$
\sum_{i=1}^{N} \int_{\Omega} a_{i}\left(x, \frac{\partial}{\partial x_{i}} T_{t}(u)\right) \cdot \frac{\partial}{\partial x_{i}} T_{t}(u) d x \leq \int_{\Omega} f(x) T_{t}(u) d x .
$$

We deduces from inequality above that

$$
\sum_{i=1}^{N} \int_{\Omega}\left|\frac{\partial}{\partial x_{i}} T_{t}(u)\right|^{p_{i}(x)} d x \leq t\|f\|_{1}, \text { for all } t>0
$$

Therefore, defining $\psi:=T_{t}(u) / t$, we have, for all $t>0$,

$$
\sum_{i=1}^{N} \int_{\Omega} t^{p_{i}(x)-1}\left|\frac{\partial}{\partial x_{i}} \psi\right|^{p_{i}(x)} d x=\frac{1}{t} \int_{\Omega}\left|\frac{\partial}{\partial x_{i}} T_{t}(u)\right|^{p_{i}(x)} d x \leq\|f\|_{1} .
$$

From the above inequality, the definition of $\alpha_{i}($.$) and (3.2), we have$

$$
\begin{gathered}
\sum_{i=1}^{N} \int_{\left\{\left|\frac{\partial}{\partial x_{i}} u\right|^{\alpha_{i}(\cdot)}>t\right\}} t^{q_{i}(x)} d x \leq \int_{\left\{\left|\frac{\partial}{\partial x_{i}} u\right|^{\alpha_{i}(\cdot)}>t\right\} \cap\{|u| \leq t\}} t^{q_{i}(x)} d x+\int_{\{|u|>t\}} t^{q_{i}(x)} d x \\
\quad \leq \int_{\{|u| \leq t\}} t^{q_{i}(x)}\left(\frac{\left|\frac{\partial}{\partial x_{i}} u\right|^{\alpha_{i}(x)}}{t}\right)^{\frac{p_{i}(x)}{\alpha_{i}(x)}} d x+M \leq\|f\|_{1}+M, \text { for all } t>0 .
\end{gathered}
$$

Proposition 3.6. Assume (1.2)-(1.6) and $f \in L^{1}(\Omega)$. Let $u$ be an entropy solution of (1.1), then

$$
\frac{1}{h} \sum_{i=1}^{N} \int_{\{|u| \leq h\}}\left|\frac{\partial}{\partial x_{i}} T_{h}(u)\right|^{p_{i}(x)} d x \leq M
$$

for every $h>0$, with $M$ a positive constant. More precisely, there exists $D>0$ such that

$$
\text { meas }\{|u|>h\} \leq D^{P_{-}^{-}} \frac{1+h}{h^{P_{-}^{-}}} .
$$

Proof. Taking $\varphi=0$ in the entropy inequality (3.1) and using (1.5), we obtain

$$
\sum_{i=1}^{N} \int_{\{|u| \leq h\}}\left|\frac{\partial}{\partial x_{i}} T_{h}(u)\right|^{p_{i}(x)} d x \leq h\|f\|_{1} \leq M h
$$

for all $h>0$.

Next,

$$
\sum_{i=1}^{N} \int_{\{|u| \leq h\}}\left|\frac{\partial}{\partial x_{i}} T_{h}(u)\right|^{p_{i}(x)} d x \leq M h \Rightarrow \sum_{i=1}^{N} \int_{\{|u| \leq h\}}\left|\frac{\partial}{\partial x_{i}} T_{h}(u)\right|^{P_{-}^{-}} d x \leq C(1+h) .
$$


We can write the above inequality as

$$
\sum_{i=1}^{N}\left\|\frac{\partial}{\partial x_{i}} T_{h}(u)\right\|_{P_{-}^{-}}^{P_{-}^{-}} \leq C(1+h) \text { or }\left\|T_{h}(u)\right\|_{W_{0}^{1, P_{-}^{-}}(\Omega)} \leq[C(1+h)]^{\frac{1}{P_{-}^{-}}} .
$$

By the Poincaré inequality in constant exponent, we obtain

$$
\left\|T_{h}(u)\right\|_{L^{P_{-}^{-}}(\Omega)} \leq D(1+h)^{\frac{1}{P_{-}^{-}}} .
$$

The above inequality imply that

$$
\int_{\Omega}\left|T_{h}(u)\right|^{P_{-}^{-}} d x \leq D^{P_{-}^{-}}(1+h),
$$

from which we obtain

$$
\text { meas }\{|u|>h\} \leq D^{P_{-}^{-}} \frac{1+h}{h^{P_{-}^{-}}} .
$$

* Step 2. Uniqueness of entropy solution. The proof of uniqueness of entropy solutions follow the technics by Bénilan et $a l[3]$ (see also [25]). Let $h>0$ and $u, v$ two entropy solutions of (1.1). We write the entropy inequality (3.1) corresponding to the solution $u$, with $T_{h} v$ as test function, and to the solution $v$, with $T_{h} u$ as test function. Upon addition, we get

$$
\left\{\begin{array}{l}
\int_{\left\{\left|u-T_{h} v\right| \leq t\right\}} \sum_{i=1}^{N} a_{i}\left(x, \frac{\partial}{\partial x_{i}} u\right) \cdot \frac{\partial}{\partial x_{i}}\left(u-T_{h} v\right) d x+ \\
\int_{\left\{\left|v-T_{h} u\right| \leq t\right\}} \sum_{i=1}^{N} a\left(x, \frac{\partial}{\partial x_{i}} v\right) \cdot \frac{\partial}{\partial x_{i}}\left(v-T_{h} u\right) d x \\
\leq \int_{\Omega} f(x)\left(T_{t}\left(u-T_{h} v\right)+T_{t}\left(v-T_{h} u\right)\right) d x .
\end{array}\right.
$$

Define

$$
E_{1}:=\{|u-v| \leq t,|v| \leq h\}, E_{2}:=E_{1} \cap\{|u| \leq h\} \text {, and } E_{3}:=E_{1} \cap\{|u|>h\} .
$$


We start with the first integral in (3.3). By (1.5), we have

$$
\begin{aligned}
& \iint_{\left\{\left|u-T_{h} v\right| \leq t\right\}} \sum_{i=1}^{N} a\left(x, \frac{\partial}{\partial x_{i}} u\right) \cdot \frac{\partial}{\partial x_{i}}\left(u-T_{h} v\right) d x= \\
& \int_{\left\{\left|u-T_{h} v\right| \leq t\right\} \cap(\{|v| \leq h\} \cup\{|v|>h\})} \sum_{i=1}^{N} a\left(x, \frac{\partial}{\partial x_{i}} u\right) \cdot \frac{\partial}{\partial x_{i}}\left(u-T_{h} v\right) d x= \\
& \int_{\left\{\left|u-T_{h} v\right| \leq t,|v| \leq h\right\}} \sum_{i=1}^{N} a\left(x, \frac{\partial}{\partial x_{i}} u\right) \cdot \frac{\partial}{\partial x_{i}}\left(u-T_{h} v\right) d x+ \\
& \int_{\left\{\left|u-T_{h} v\right| \leq t,|v|>h\right\}} \sum_{i=1}^{N} a\left(x, \frac{\partial}{\partial x_{i}} u\right) \cdot \frac{\partial}{\partial x_{i}}\left(u-T_{h} v\right) d x \\
& =\int_{\{|u-v| \leq t,|v| \leq h\}} \sum_{i=1}^{N} a\left(x, \frac{\partial}{\partial x_{i}} u\right) \cdot \frac{\partial}{\partial x_{i}}(u-v) d x+\int_{\left\{\left|u-T_{h} v\right| \leq t,|v|>h\right\}} \sum_{i=1}^{N} a\left(x, \frac{\partial}{\partial x_{i}} u\right) \cdot \frac{\partial}{\partial x_{i}} u d x \\
& \geq \int_{\{|u-v| \leq t,|v| \leq h\}} \sum_{i=1}^{N} a\left(x, \frac{\partial}{\partial x_{i}} u\right) \cdot \frac{\partial}{\partial x_{i}}(u-v) d x=\int_{E_{1}} \sum_{i=1}^{N} a\left(x, \frac{\partial}{\partial x_{i}} u\right) \cdot \frac{\partial}{\partial x_{i}}(u-v) d x \\
& =\int_{E_{1} \cap(\{|u| \leq h\} \cup\{|u|>h\})} \sum_{i=1}^{N} a\left(x, \frac{\partial}{\partial x_{i}} u\right) \cdot \frac{\partial}{\partial x_{i}}(u-v) d x=\int_{E_{2}} \sum_{i=1}^{N} a\left(x, \frac{\partial}{\partial x_{i}} u\right) \cdot \frac{\partial}{\partial x_{i}}(u-v) d x \\
& +\int_{E_{3}} \sum_{i=1}^{N} a\left(x, \frac{\partial}{\partial x_{i}} u\right) \cdot \frac{\partial}{\partial x_{i}}(u-v) d x=\int_{E_{2}} \sum_{i=1}^{N} a\left(x, \frac{\partial}{\partial x_{i}} u\right) \cdot \frac{\partial}{\partial x_{i}}(u-v) d x+ \\
& \int_{E_{3}} \sum_{i=1}^{N} a\left(x, \frac{\partial}{\partial x_{i}} u\right) \cdot \frac{\partial}{\partial x_{i}} u d x-\int_{E_{3}} \sum_{i=1}^{N} a\left(x, \frac{\partial}{\partial x_{i}} u\right) \cdot \frac{\partial}{\partial x_{i}} v d x \\
& \geq \int_{E_{2}} \sum_{i=1}^{N} a\left(x, \frac{\partial}{\partial x_{i}} u\right) \cdot \frac{\partial}{\partial x_{i}}(u-v) d x-\int_{E_{3}} \sum_{i=1}^{N} a\left(x, \frac{\partial}{\partial x_{i}} u\right) \cdot \frac{\partial}{\partial x_{i}} v d x
\end{aligned}
$$


Using (1.3) and (2.1), we estimate the last integral in (3.4) as follows

$$
\left\{\begin{array}{l}
\left|\int_{E_{3}} \sum_{i=1}^{N} a\left(x, \frac{\partial}{\partial x_{i}} u\right) \cdot \frac{\partial}{\partial x_{i}} v d x\right| \leq C_{1} \int_{E_{3}} \sum_{i=1}^{N}\left(j_{i}(x)+\left|\frac{\partial}{\partial x_{i}} u\right|^{p_{i}(x)-1}\right)\left|\frac{\partial}{\partial x_{i}} v\right| d x \\
\leq C \sum_{i=1}^{N}\left(|j|_{p_{i}^{\prime}(.)}+\left.\left.|| \frac{\partial}{\partial x_{i}} u\right|^{p_{i}(x)-1}\right|_{p_{i}^{\prime}(.),\{h<|u| \leq h+t\}}\right)\left|\frac{\partial}{\partial x_{i}} v\right|_{p_{i}(.),\{h-t<|v| \leq h\}},
\end{array}\right.
$$

where $\left.\left.|| \frac{\partial}{\partial x_{i}} u\right|^{p_{i}(x)-1}\right|_{p_{i}^{\prime}(.),\{h<|u| \leq h+t\}}=\left\|\left|\frac{\partial}{\partial x_{i}} u\right|^{p_{i}(x)-1}\right\|_{L^{p_{i}^{\prime}(.)}(\{h<|u| \leq h+t\})}$.

For all $i=1, \ldots, N$, the quantity $\left(\left|j_{i}\right|_{p_{i}^{\prime}(.)}+\left.\left.|| \frac{\partial}{\partial x_{i}} u\right|^{p_{i}(x)-1}\right|_{p_{i}^{\prime}(.),\{h<|u| \leq h+t\}}\right)$ is finite, since $u \in W_{0}^{1, \vec{p}(.)}(\Omega)$ and $j_{i} \in L^{p_{i}^{\prime}(\cdot)}(\Omega)$; then by Proposition 3.6, the last expression converges to zero as $h$ tends to infinity. Therefore, from (3.4) and (3.5), we obtain

$$
\int_{\left\{\left|u-T_{h} v\right| \leq t\right\}} \sum_{i=1}^{N} a\left(x, \frac{\partial}{\partial x_{i}} u\right) \cdot \frac{\partial}{\partial x_{i}}\left(u-T_{h} v\right) d x \geq I_{h}+\int_{E_{2}} \sum_{i=1}^{N} a\left(x, \frac{\partial}{\partial x_{i}} u\right) \cdot \frac{\partial}{\partial x_{i}}(u-v) d x,
$$

where $I_{h}$ converges to zero as $h$ tends to infinity. We may adopt the same procedure to treat the second term in (3.3) to obtain

$$
\int_{\left\{\left|v-T_{h} u\right| \leq t\right\}} \sum_{i=1}^{N} a\left(x, \frac{\partial}{\partial x_{i}} v\right) \cdot \frac{\partial}{\partial x_{i}}\left(v-T_{h} u\right) d x \geq J_{h}-\int_{E_{2}} \sum_{i=1}^{N} a\left(x, \frac{\partial}{\partial x_{i}} v\right) \cdot \frac{\partial}{\partial x_{i}}(u-v) d x,
$$

where $J_{h}$ converges to zero as $h$ tends to infinity.

Next, consider the right-hand side of inequality (3.3). Noting that

$$
T_{t}\left(u-T_{h} v\right)+T_{t}\left(v-T_{h} u\right)=0 \text { in }\{|u| \leq h,|v| \leq h\} ;
$$

we obtain

$$
\begin{aligned}
& \left|\int_{\Omega} f(x)\left(T_{t}\left(u-T_{h} v\right)+T_{t}\left(v-T_{h} u\right)\right) d x\right|= \\
& \left|\int_{\{|u|>h\}} f(x)\left(T_{t}\left(u-T_{h} v\right)+T_{t}\left(v-T_{h} u\right)\right) d x+\int_{\{|u| \leq h\}} f(x)\left(T_{t}\left(u-T_{h} v\right)+T_{t}\left(v-T_{h} u\right)\right) d x\right|= \\
& \left|\int_{\{|u|>h\}} f(x)\left(T_{t}\left(u-T_{h} v\right)+T_{t}\left(v-T_{h} u\right)\right) d x+\int_{\{|u| \leq h,|v|>h\}} f(x)\left(T_{t}\left(u-T_{h} v\right)+T_{t}\left(v-T_{h} u\right)\right) d x\right| \\
& \leq 2 t\left(\int_{\{|u|>h\}}|f| d x+\int_{\{|v|>h\}}|f| d x\right) .
\end{aligned}
$$

According to Proposition 3.6, both meas $\{|u|>h\}$ and meas $\{|v|>h\}$ tend to zero as $h$ goes to infinity, then by the inequality above, the right-hand side of inequality (3.3) tends to zero as $h$ 
goes to infinity. From this assertion, (3.3), (3.6) and (3.7), we obtain, letting $h \rightarrow+\infty$,

$$
\int_{\{|u-v| \leq t\}} \sum_{i=1}^{N}\left(a\left(x, \frac{\partial}{\partial x_{i}} u\right)-a\left(x, \frac{\partial}{\partial x_{i}} v\right)\right) \cdot \frac{\partial}{\partial x_{i}}(u-v) d x \leq 0, \text { for all } t>0
$$

By assertion (1.4), we conclude that $\frac{\partial}{\partial x_{i}} u=\frac{\partial}{\partial x_{i}} v$, for all $i=1, . ., N$ a.e. in $\Omega$.

We deduce that

$$
\|u-v\|_{\vec{p}(.)}=\sum_{i=1}^{N}\left|\frac{\partial}{\partial x_{i}} u-\frac{\partial}{\partial x_{i}} v\right|_{p_{i}(.)}=0,
$$

and hence $u=v$, a.e. in $\Omega$.

* Step 3. Existence of entropy solutions. Let $\left(f_{n}\right)_{n}$ be a sequence of bounded functions, strongly converging to $f \in L^{1}(\Omega)$ and such that

$$
\left\|f_{n}\right\|_{1} \leq\|f\|_{1} \text {, for all } n \text {. }
$$

We consider the problem

$$
\left\{\begin{array}{l}
-\sum_{i=1}^{N} \frac{\partial}{\partial x_{i}} a_{i}\left(x, \frac{\partial}{\partial x_{i}} u_{n}\right)=f_{n} \text { in } \Omega \\
u_{n}=0 \text { on } \partial \Omega .
\end{array}\right.
$$

It follows from Theorem 2.3 (cf. [15]) that problem (3.9) has a unique weak energy solution $u_{n} \in W_{0}^{1, \vec{p}(.)}(\Omega)$ since $f_{n} \in L^{\infty}(\Omega)$. Our interest is to prove that these approximated solutions $u_{n}$ tend, as $n$ goes to infinity, to a measurable function $u$ which is an entropy solution of the limit problem (1.1). To this end, we derive a priori estimates for $u_{n}$ and $\frac{\partial}{\partial x_{i}} u_{n}$ in the Marcinkiewicz spaces $\mathcal{M}^{q^{*}}$ and $\mathcal{M}^{p_{i}^{-}} q / \bar{p}$ where $q^{*}$ and $\bar{p}$ are defined in (1.6) and (1.7).

Let $\gamma>0$, denote $T_{\gamma}$ the corresponding truncation function. Note that

$$
D T_{\gamma}(r)=\left\{\begin{array}{c}
1 \text { if }|r|<\gamma \\
0 \text { if }|r|>\gamma
\end{array}\right.
$$

In particular, we have

$$
a_{i}(x, \xi) D T_{\gamma}(r) \xi \geq a_{i}(x, \xi) \xi \chi_{\{|r|<\gamma\}}, \text { for all } i=1, \ldots, N
$$

Lemma 3.7. There exists a constant $c$, not depending on $n$, such that

$$
\sum_{i=1}^{N} \int_{\left\{\left|u_{n}\right| \leq \gamma\right\}}\left|\frac{\partial u_{n}}{\partial x_{i}}\right|^{p_{i}^{-}} d x \leq c(\gamma+1), \text { for all } \gamma>0
$$


Proof. Inserting $\varphi=T_{\gamma}\left(u_{n}\right)$ into (2.2), we have

$$
\int_{\Omega} \sum_{i=1}^{N} a_{i}\left(x, \frac{\partial}{\partial x_{i}} u_{n}\right) D T_{\gamma}\left(u_{n}\right) \frac{\partial}{\partial x_{i}} u_{n} d x=\int_{\Omega} f_{n} T_{\gamma}\left(u_{n}\right) d x .
$$

Using (3.10) and the coercivity condition (1.5), we obtain (3.11).

Lemma 3.8. There exists a constant $C$, not depending on $n$, such that

$$
\left\|u_{n}\right\|_{\mathcal{M}^{q^{*}}(\Omega)} \leq C
$$

and

$$
\left\|\frac{\partial u_{n}}{\partial x_{i}}\right\|_{\mathcal{M}^{p^{-} q / \bar{p}}(\Omega)} \leq C, \text { for all } i=1, \ldots, N .
$$

Proof. The result of Lemma 3.8 is a direct consequence of Lemma 3.7 (cf. [2, proof of Lemma 3.3]).

In view of Lemma 3.8 and following [2] ( see also [4, Lemma A.2]), we deduce that $u_{n}$ is uniformly bounded in $L^{k_{0}}(\Omega)$ for some $k_{0}<q^{*}$ with $k_{0}>p_{i}^{-} q / \bar{p}$ and $\frac{\partial u_{n}}{\partial x_{i}}$ is uniformly bounded in $L^{k_{i}}(\Omega)$ for some $k_{i}>1$ with $p_{i}^{-}-1<k_{i}<p_{i}^{-} q / \bar{p}$, for all $i=1, \ldots, N$. From this, we get that $u_{n}$ is uniformly bounded in the isotropic Sobolev space $W_{0}^{1, k_{\min }}(\Omega)$, where $k_{\min }=\min \left(k_{1}, \ldots, k_{N}\right)$. Consequently, we can assume without loss of generality (see also [2, Lemma 3.4]) that as $n \rightarrow 0$,

$$
\left\{\begin{array}{l}
u_{n} \rightarrow u \text { a.e in } \Omega \text { and in } L^{k_{\min }}(\Omega) \\
u_{n} \rightarrow u \text { in } W_{0}^{1, k_{\min }}(\Omega) \\
\frac{\partial u_{n}}{\partial x_{i}} \rightarrow \frac{\partial u}{\partial x_{i}} \text { in } L^{1}(\Omega), \text { for all } i=1, \ldots, N . \\
a_{i}\left(x, \frac{\partial u_{n}}{\partial x_{i}}\right) \rightarrow a_{i}\left(x, \frac{\partial u}{\partial x_{i}}\right) \text { a.e in } \Omega \text { and in } L^{1}(\Omega), \text { for all } i=1, \ldots, N .
\end{array}\right.
$$

Now, fix $t>0, \varphi \in W_{0}^{1, \vec{p}(.)}(\Omega) \cap L^{\infty}(\Omega)$, and choose $T_{t}\left(u_{n}-\varphi\right)$ as a test function in (2.2), with $u$ replaced by $u_{n}$ to obtain

$$
\int_{\Omega} \sum_{i=1}^{N} a_{i}\left(x, \frac{\partial}{\partial x_{i}} u_{n}\right) \cdot \frac{\partial}{\partial x_{i}} T_{t}\left(u_{n}-\varphi\right) d x=\int_{\Omega} f_{n}(x) T_{t}\left(u_{n}-\varphi\right) d x .
$$

Note that this choice can be made using a standard density argument. We now pass to the limit in the previous identity. For the right-hand side, the convergence is obvious since $f_{n}$ converges strongly in $L^{1}$ to $f$ and $T_{t}\left(u_{n}-\varphi\right)$ converges weakly-* in $L^{\infty}$, and a.e., to $T_{t}(u-\varphi)$.

Next, we write the left hand side as

$$
\int_{\left\{\left|u_{n}-\varphi\right| \leq t\right\}} \sum_{i=1}^{N} a_{i}\left(x, \frac{\partial}{\partial x_{i}} u_{n}\right) \cdot \frac{\partial}{\partial x_{i}} u_{n} d x-\int_{\left\{\left|u_{n}-\varphi\right| \leq t\right\}} \sum_{i=1}^{N} a_{i}\left(x, \frac{\partial}{\partial x_{i}} u_{n}\right) \cdot \frac{\partial}{\partial x_{i}} \varphi d x
$$


By (3.12), the second integral of (3.13) converges to

$$
\int_{\{|u-\varphi| \leq t\}} \sum_{i=1}^{N} a_{i}\left(x, \frac{\partial}{\partial x_{i}} u\right) \cdot \frac{\partial}{\partial x_{i}} \varphi d x .
$$

For the first integral in (3.13), as $\sum_{i=1}^{N} a_{i}\left(x, \frac{\partial}{\partial x_{i}} u_{n}\right) \cdot \frac{\partial}{\partial x_{i}} u_{n}$ is nonnegative by (1.5), we obtain by using (3.12) and Fatou's Lemma

$$
\int_{\{|u-\varphi| \leq t\}} \sum_{i=1}^{N} a_{i}\left(x, \frac{\partial}{\partial x_{i}} u\right) \cdot \frac{\partial}{\partial x_{i}} u d x \leq \lim _{n \rightarrow \infty} \inf \int_{\left\{\left|u_{n}-\varphi\right| \leq t\right\}} \sum_{i=1}^{N} a_{i}\left(x, \frac{\partial}{\partial x_{i}} u_{n}\right) \cdot \frac{\partial}{\partial x_{i}} u_{n} d x .
$$

Gathering results, we obtain

$$
\int_{\Omega} \sum_{i=1}^{N} a_{i}\left(x, \frac{\partial}{\partial x_{i}} u\right) \cdot \frac{\partial}{\partial x_{i}} T_{t}(u-\varphi) d x \leq \int_{\Omega} f(x) T_{t}(u-\varphi) d x
$$

i.e., $u$ is an entropy solution of (1.1).

Received: June, 2008. Revised: november, 2008.

\section{References}

[1] Alvino, A., Boccardo, L., Ferone, V., Orsina, L. and Trombetti, G., Existence results for non-linear elliptic equations with degenerate coercivity, Ann. Mat. Pura Appl., 182 (2003), 53-79.

[2] Bendahmane, M. and Karlsen, K.H., Anisotropic nonlinear elliptic systems with measure data and anisotropic harmonic maps into spheres, Electron. J. Differential Equations 2006, No. 46, 30 pp.

[3] Bénilan, Ph., Boccardo, L., Gallouët, T., Gariepy, R., Pierre, M. and Vazquez, J.L., An $L^{1}$-theory of existence and uniqueness of solutions of nonlinear elliptic equations, Ann. Sc. Norm. Super. Pisa, Cl. Sci., 22 (1995), 241-273.

[4] Bénilan, Ph., Brezis, H. and Crandall, M.G., A semilinear equation in $L^{1}(\mathbb{R})^{N}$, Ann. Scula. Norm. Sup. Pisa, 2 (1975), 523-555.

[5] Chen, Y., Levine, S. and Rao, M., Variable exponent, linear growth functionals in image restoration, SIAM J. Appl. Math. 66, no. 4 (2006), 1383-1406. 
[6] Diening, L., Theoretical and Numerical Results for Electrorheological Fluids, PhD. thesis, University of Frieburg, Germany, 2002.

[7] Diening, L., Riesz potential and Sobolev embeddings on generalized Lebesgue and Sobolev spaces $L^{p(.)}$ and $W^{1, p(.)}$, Math. Nachr., 268 (2004), 31-43.

[8] Edmunds, D.E. And Rakosnik, J., Density of smooth functions in $W^{k, p(x)}(\Omega)$, Proc. R. Soc. A, 437 (1992), 229-236.

[9] Edmunds, D.E. and Rakosnik, J., Sobolev embeddings with variable exponent, Studia Math., 143 (2000), 267-293.

[10] Edmunds, D.E. And Rakosnik, J., Sobolev embeddings with variable exponent, II, Math. Nachr., 246-247 (2002), 53-67.

[11] El Hamidi, A., Existence results to elliptic systems with nonstandard growth conditions, J. Math. Anal. Appl., 300 (2004), 30-42.

[12] Fan, X. And Zhang, Q., Existence of solutions for $p(x)$-Laplacian Dirichlet problem, Nonlinear Anal., 52 (2003), 1843-1852.

[13] Harjulehto, P., Hästö, P., Koskenova, M. and Varonen, S., The Dirichlet energy integral and variable exponent Sobolev spaces with zero boundary values, Potential Anal., 25 (2006), 79-94.

[14] Hudzik, H., On generalized Orlicz-Sobolev space, Funct. Approximatio Comment. Math., 4 (1976), 37-51.

[15] Koné, B., Ouaro, S. and Traoré, S., Weak solutions for anisotropic nonlinear elliptic equations with variable exponent, Electron J. Differ. Equ., 144 (2009), 1-11.

[16] Kovacik, O. And Rakosnik, J., On spaces $L^{p(x)}$ and $W^{1, p(x)}$, Czech. Math. J., 41 (1991), 592-618.

[17] Leray, J. And Lions, J.L., Quelques résultats de Visik sur les problèmes elliptiques nonlinéaires par les méthodes de Minty et Browder, Bull. Soc. Math. France., 93 (1965), 97-107.

[18] Minallescu, M., Pucci, P. and Radulescu, V., Eigenvalue problems for anisotropic quasilinear elliptic equations with variable exponent. J. Math. Anal. Appl., 340 (2008), no. $1,687-698$.

[19] Minailescu, M. and Radulescu, V., A multiplicity result for a nonlinear degenerate problem arising in the theory of electrorheological fluids, Proc. R. Soc. A, 462 (2006), 26252641.

[20] MusielaK, J., Orlicz Spaces and modular spaces, Lecture Notes in Mathematics, 1034 (1983), springer, Berlin. 
[21] OrLicz, W., Über konjugierte Exponentenfolgen, Studia Math., 3 (1931), 200-212.

[22] Pfeiffer, C., Mavroidis, C., Bar-Cohen, Y. and Dolgin, B., Electrorheological fluid based force feedback device, in Proc. 1999 SPIE Telemanipulator and Telepresence Technologies VI Conf. (Boston, MA), 3840 (1999), pp. 88-99.

[23] Rajagopal, K.R. And Ruzicka, M., Mathematical modelling of electrorheological fluids, Continuum Mech. Thermodyn., 13 (2001), 59-78.

[24] Ruzicka, M., Electrorheological fluids: modelling and mathematical theory, Lecture Notes in Mathematics 1748, Springer-Verlag, Berlin, 2000.

[25] Sanchon, M. and Urbano, J.M., Entropy solutions for the $p(x)$-Laplace Equation, Trans. Amer. Math. Soc., 361 (2009), no 12, 6387-6405.

[26] Sharapudinov, I., On the topology of the space $L^{p(t)}([0,1])$, Math. Zametki, 26 (1978), 613-632.

[27] Tsenov, I.V., Generalization of the problem of best approximation of a function in the space $L^{s}$, Uch. Zap. Dagestan Gos. Univ., 7 (1961), 25-37.

[28] Winslow, W.M., Induced Fibration of Suspensions, J. Applied Physics, 20 (1949), 11371140. 